\title{
Counting the costs of ankylosing spondylitis
}

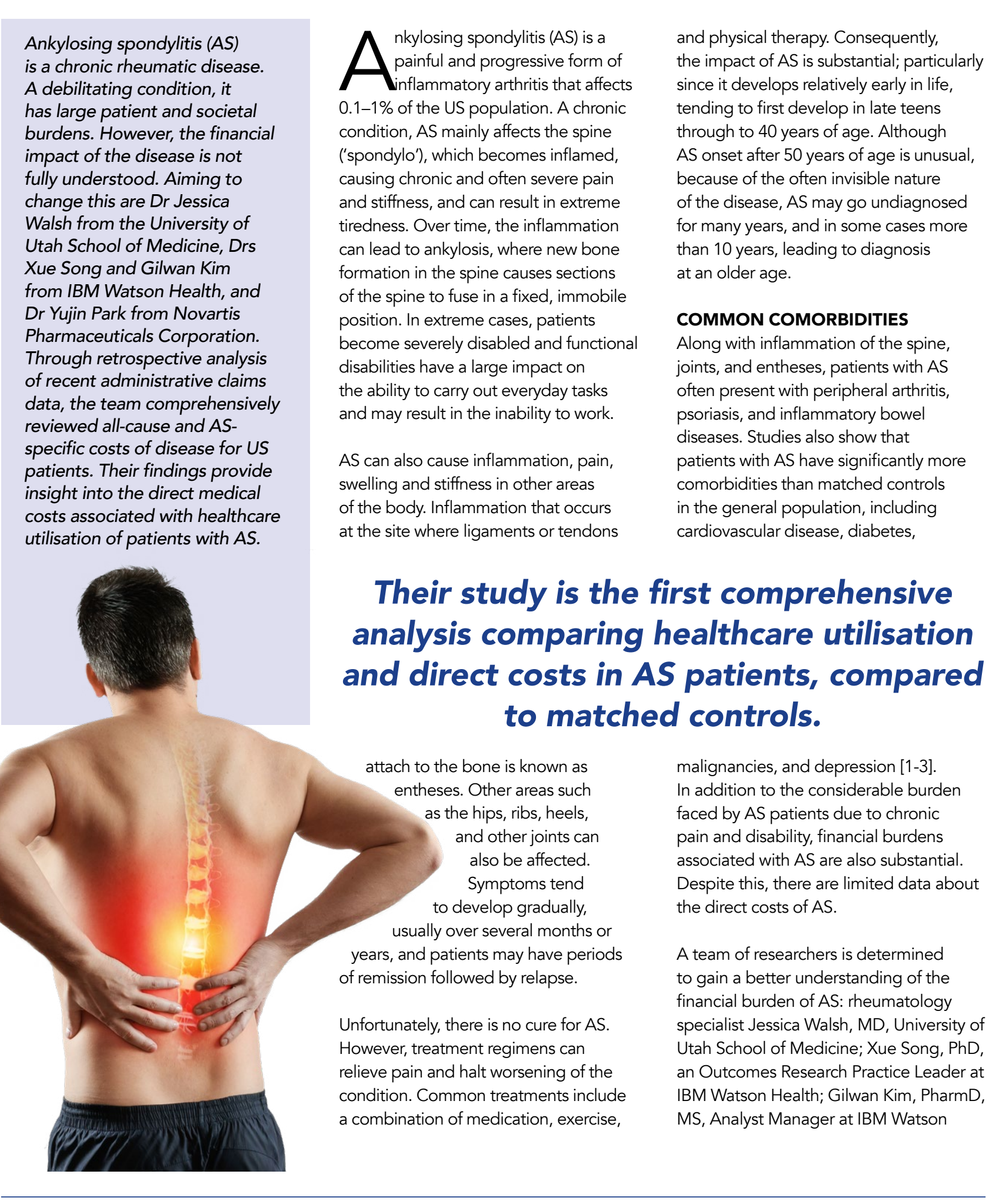

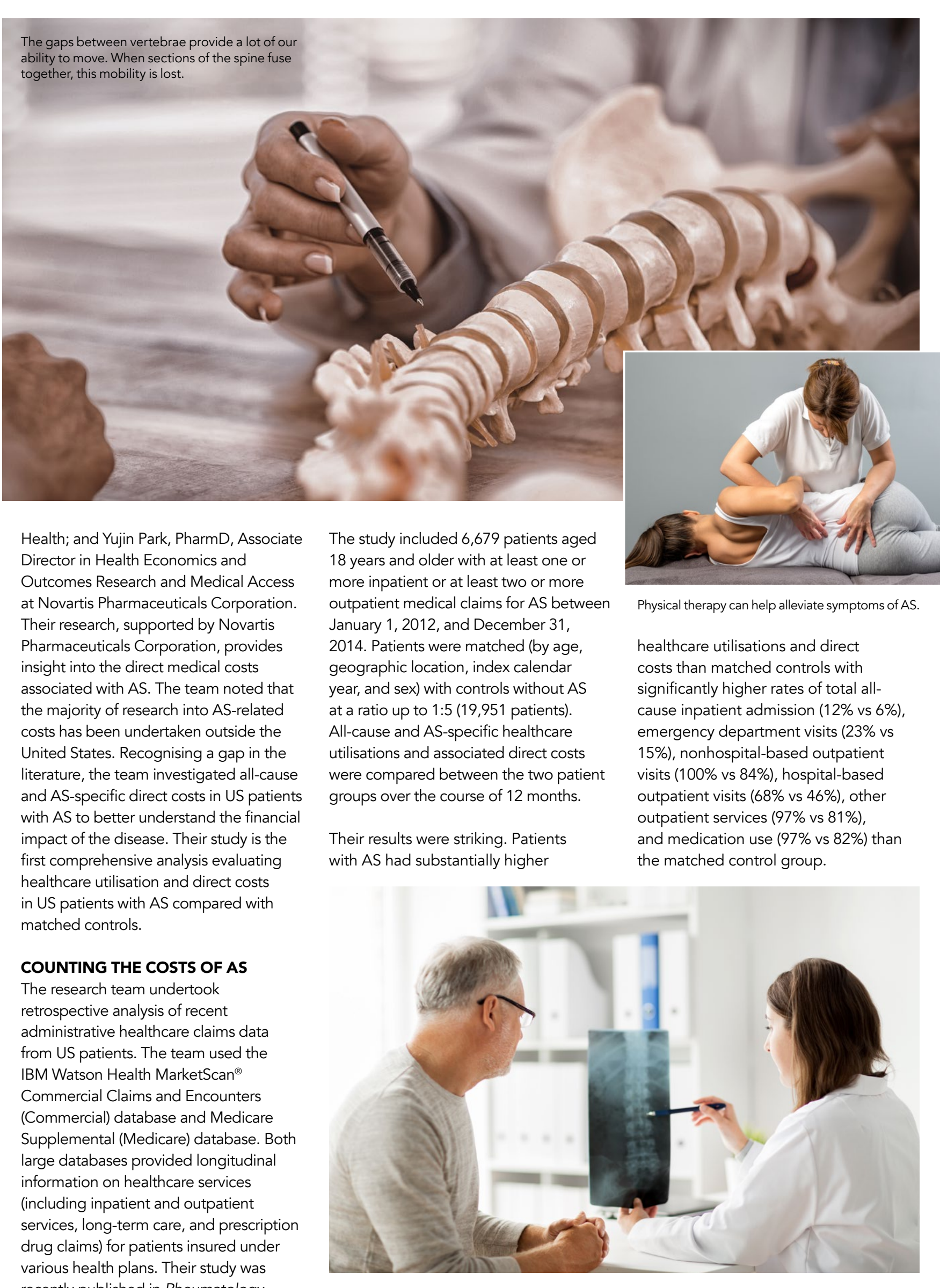

durisus for recently published in Rheumatology and Therapy. 
Notably, patients with AS had a tenfoldcost than matched controls (\$24,978 vs \$2,139 per patient per year). Higher costs associated with AS were largely a result of increased medical outpatient senvices mean cost $\$ 13,220$ vs $\$ 4,602$, per patien per year) and outpatient pharmacy costs (mean cost $\$ 14,074$ vs $\$ 1,737$, per patient per year), as illustrated in the figure opposite. Outpatient pharmacy costs associated with AS included use of biologic therapies, other AS-related medications (e.g. anti-inflammatory drug, antirheumatic drugss), antihypertensives, and antidepressants.

The research team suggests that the presence of common comorbidities and costs obsen in petients with AS compared to matched controls. Their analysis showed that patients with AS had significantly higher rates of cardiovascular disease, depression, malignancies, osteoporosis, sleep apnoea, and spinal fracture, as well as inflammatory bowe disease and psoriasis than matched controls. These comorbidities require additional medications, treatments and complicate AS management, leading to increased costs. However, as the authors note: "Further research is needed to fully determine the potentia
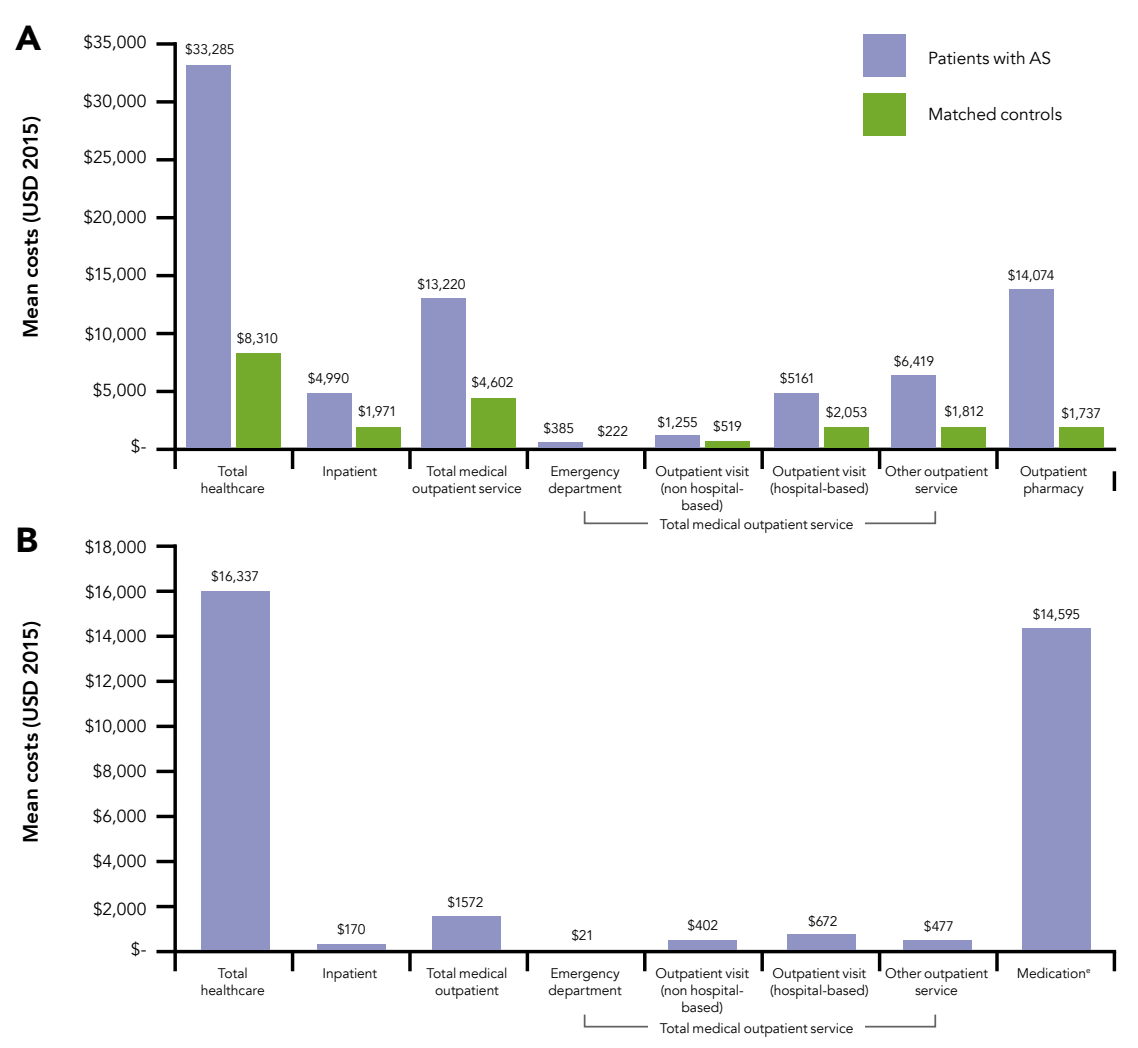
A (top): Mean direct healthcare costs per patient per year over a 12-month follow-up period. All-cause
healthcare costs for patients with AS and matched controls. Note how the cost for patients with AS is consistently higher than for the matched controls.
$B$ (bottom): AS-related healthcare costs for patients with AS

AS and comorbidities and the role

and costs could not be determined in the of inflammation in the development of comorbidities in patients with AS." The team also noted that effects of severity of disease and other risk factors (e.g. obesty

The research of this team of colleagues fills a gap in the body of knowledge on AS comorbidities and costs of care for AS patients in the USA.

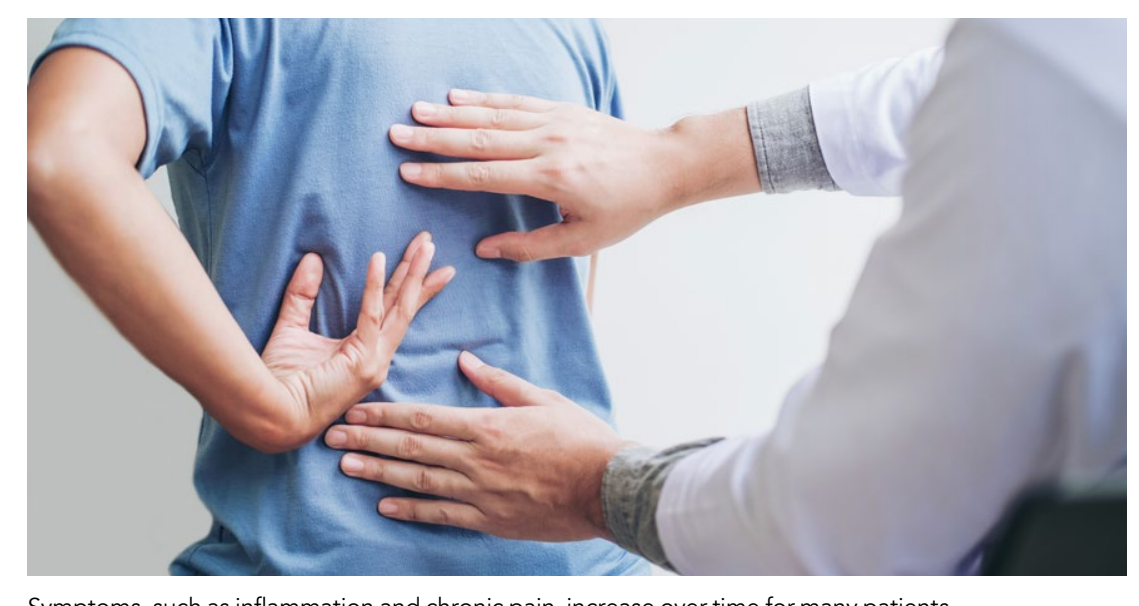

CONCLUSION

The research of this team of colleagues fills a gap in the body of knowledge on AS comorbidities and costs of care for US pepresent with $A S$ : in addition to its physical psychological and social burden, the current study highlights the high economic burden of the healthcare needs of AS patients. Findings from their descriptive analysis using large administrative claims databases provide key insights into the direct medical costs associated with healthcare utilisation in patients with AS across the United States. The total financia burden of AS could not be determined in the current study because indirect costs f (n) Wed a burden of AS from the individul petiont standpoint and society perspective would be beneficial.

\section{Behind the Research}

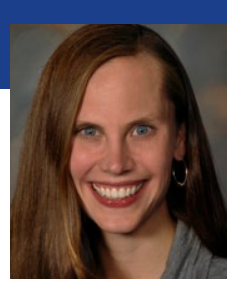

Dr Jessica A. Walsh

E: Jessica.walsh@hsc.utah.edu

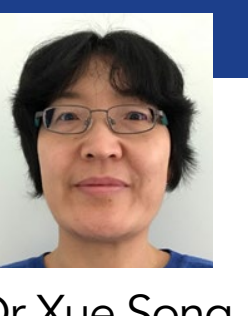

Dr Xue Song

E: songx@us.ibm.com

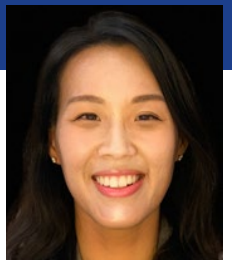

Dr Gilwan Kim

E: kimgi@us.ibm.com

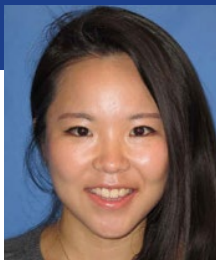

Dr Yujin Park

E: Jina.park@novartis.com

\section{Research Objectives}

The team's work has unveiled the direct costs of healthcare for patients with ankylosing spondylitis.

\section{Detail}

Jessica A. Walsh

Universti of Utah School of Medicine and Salt Lake City Affairs Medical Center, Salt Lake City, UT

Xue Song

IBM Watson Health, Cambridge MA

Gilwan Kim

BM Watson Health, Cambridge, MA

Yujin Park

East Hanover, NJ

Jessica Walsh, MD, is an Instructor at the University of Utah School of Medicine and George E. Wahlen Veteran Affairs Center As a rheumatologist, her clinical interests include spondyloarthritis and psoriatic diseases.

Xue Song, PhD, is an Outcomes Research Practice Leader at IBM Watson Health.

Gilwan Kim, PharmD, MS, is an Analyst Manager at IBM Watson Health.

Yujin Park, PharmD, is an Associate Director in Health conomics and Outcomes Research and Medical Access t Novartis Pharmaceuticas

Funding

This study was sponsored by Novartis Pharmaceuticals Corporation, East Hanover, NJ.

DrWalsh is a consultant for Novartis Pharmaceuticals Corporation.

Dr Song and Dr Kim are employees of IBM Watson Health.

Dr Park is an employee of Novartis

\section{References}

1 Walsh JA, Song X, Kim G, Park Y. (2018). Healthcare utilization and direct costs in patients with ankylosing spondylitis using a large US administrative claims database. Rheumatol Ther. 5(2):463-474. doi: 10.1007/ s40744-018-0124-4.

2 Walsh JA, Song X, Kim G, Park Y. (2018). Evaluation of the comorbidity burden in patients with ankylosing spondylitis using a large US administrative claims data set. Clin Rheumatol. 37(7):1869-1878. doi: 10.1007/ s10067-018-4086-2.

3 Walsh JA, Song X, Kim G, Park Y. (2018). Evaluation of the comorbidity burden in patients with ankylosing spondylitis treated with tumour necrosis factor inhibitors Health Serv Res. 9(2):115-121. doi: 10.1111/jphs.12212.

\section{Personal Response}

Your research provides insight into the cost of the healthcare needs of patients with AS. What's next for your work?

AS, This study looked at direct costs associated with AS, but studies that evaluate societal and indirect costs, such as work productivity, disability, and caregiver costs, will provide a more complete picture of the economic US healthcare system because previous studies have shown indirect costs associated with AS to be higher than direct costs. In addition, as the diagnostic delay of AS is one of the biggest challenges in optimising care, further research quantifying the cost of delayed AS help to raise the importance of this issue. 